\title{
An Analysis of Sociodemographic and Clinical Characteristics in Children and Adolescents Diagnosed with Childhood Onset Speech Fluency Disorder
}

\author{
Börte Gürbüz Özgür'1, Erdoğan Özgür² \\ 1 Muğla Sttkı Koçman University Training and Research Hospital, Child and Adolescent Psychiatry Clinic, Muğla, TURKEY \\ ORCID: 0000-0002-9176-7359 \\ 2 Muğla Sitkı Koçman University, Faculty of Medicine, Department of ENT, Muğla, Turkey \\ ORCID: 0000-0003-2494-4244
}

\begin{abstract}
Objective: Childhood onset speech fluency disorder (stuttering) is a communication disorder beginning in childhood, and is characterised by interruption to speech flow, sound prolongations and pauses produced by repetition of a particular sound or word. The aim of this study was to investigate the sociodemographic characteristics, comorbid psychiatric diagnoses and other variables that are associated with stuttering.
\end{abstract}

Methods: Sixty-four children and adolescents aged under 18 years and attending the child and adolescent psychiatry or otorhinolaryngology (ENT) outpatient clinic between November 2017 and June 2019 were enrolled in the study. ENT examination was carried out. A sociodemographic questionnaire was also administered to the participants. The psychiatric evaluation of the patients was performed according to DSM- 5 criteria.

Results: The mean age was $7.36 \pm 3.76$ years. $70.3 \%$ of the cases were male. The mean age at onset of stuttering was $4.85 \pm 2.34$ years and the average stuttering duration was 2.31 years. In $31.3 \%$ of cases a life stressor had preceded stuttering, $21.9 \%$ had a positive family history, and $40.6 \%$ had comorbid psychiatric morbidity. The most common comorbid psychiatric diagnoses were specific learning disorder, speech sound disorder and attention deficit hyperactivity disorder.

Conclusion: According to the results obtained, stuttering was 2.3 times more common in males, and $68.7 \%$ of all cases were below the age of 5 years. The most common psychiatric diagnoses found in cases of stuttering are within the neurodevelopmental disorders grouping. Since psychiatric diagnoses occur at such a high frequen$c y$, it is important to address this comorbidity when treating stuttering.

Keywords: Child, language, learning disorder, speech, stuttering.
Childhood onset speech fluency disorder (stuttering) is a communication disorder beginning in childhood, and characterised by interruptions to speech flow, sound prolongations and pauses produced by repetition of a particular sound or word. Overall, $95 \%$ of stuttering cases begin before the age of 5 years. ${ }^{[1]}$ Although it is estimated that the lifetime prevalence of stuttering is only around $0.3-1 \%$, it has been ascertained that 5 out of 100 people do actually have a stuttering problem at some period in their lives. ${ }^{[2,3]}$ When undiagnosed cases, or those which have already re- 
mitted spontaneously are taken into account, it is thought that the true rate will be higher than that previously reported. ${ }^{[4]}$ Some investigators have noted that the frequency of stuttering in children varies according to race or ethnicity. ${ }^{[5]}$ In addition, it is more frequent to observe stuttering in children with genetic syndromes, such as Down Syndrome or Fragile $\mathrm{X}^{[6]}$ Stuttering can appear in different guises: it can be in the form of repeating a particular sound or word, prolonging a sound, interjecting, pausing within words, filled or unfilled pauses in speech, circumlocutions, excessive word stress and repetition of words consisting of a single syllable. ${ }^{[7]}$ These clinical findings and symptoms are listed amongst the diagnostic criteria for "childhood onset speech fluency disorder", within the category of neuro-developmental disorders in the Diagnostic and Statistical Manual of Mental Disorders-5 (DSM 5). ${ }^{[7]}$ The intensity and frequency of stuttering may vary in different contexts, or depending on whom a child is talking to. Additionally, secondary behaviours may also be observed, such as clearing the throat, repeating the "um" sound, blinking the eyes, or shaking the head when initiating speech. These behaviours may also be linked to the avoidance of speaking. Stuttering may be classified into developmental, neurogenic, and psychogenic types. ${ }^{[8]}$ Although DSM 5 does not include such a system of classification, it does acknowledge that neurological factors should be ruled out as a cause when making a diagnosis of stuttering, and stuttering should be coded "adult-onset" when it starts in the post-adolescence period. ${ }^{[7]}$ Childhood stuttering is a multi-factorial diagnosis, encompassing genetic, psychological, neurological and behavioural characteristics, and each of the factors causing stuttering should be focused on in turn. ${ }^{[9-12]}$ Stuttering in adults is usually related to an underlying neurogenic or psychogenic condition. ${ }^{[13]}$ In studies which have investigated the family history of stuttering children, the rate of a positive family history ranges between 20 and $74 \% .{ }^{[14]} \mathrm{Al}-$ though the high frequency of a positive family history in sufferers suggests a genetic basis, no chromosomal anomaly or model for how the disorder may be inherited has yet been identified. ${ }^{[14]}$ Amongst some of the neurogenic factors implicated in the aetiology of the disorder, differences in the relative volumes of grey and white matter in the brain, differences in neuronal pathways, atypical lateralisation of functions to the opposite hemisphere and increased white matter connections within the right hemisphere have been described. ${ }^{[15,16]}$ In the psychiatric evaluation of children who stutter, behavioural problems, various fears or other psychosocial stressors preceding the onset of stuttering have been observed. ${ }^{[17]}$ In addition, it has been reported that a lack of self-confidence, introversion and obsessional character traits are encountered more frequently in subjects who stutter. Shyness, being ridiculed, inhibitions and worries can adversely impact the lives of children who stutter. $^{[18]}$

In this study, the aim was to evaluate the sociodemographic characteristics and comorbid psychiatric diagnoses of children and adolescents diagnosed with stuttering.

\section{Materials and Methods}

The study was granted ethical approval by the Human Research Ethics Committee of Mugla Sıtkı Koçman University. The design was a retrospective chart review study. The participants in the study consisted of 64 children and adolescents under the age of 18 diagnosed with stuttering at the Child and Adolescent Psychiatry and Otorhinolaryngology outpatient clinics in Mugla S1tkı Koçman University Training and Research Hospital. All the cases underwent examination by a child psychiatrist. The age of onset of stuttering, its duration, the existence of triggering factors, any family history, developmental characteristics, comorbid psychiatric diagnoses and whether the subjects received speech therapy or special training were investigated, in addition to their sociodemographic characteristics. The diagnosis of stuttering was made in accordance with DSM-5 diagnostic criteria, following detailed clinical examination. Cognitive function and level of intelligence were assessed by means of age-appropriate tests (i.e. the Ankara Development Test Inventory, Denver Developmental Screening Test [Denver II], Wechsler Intelligence Scale for Children, [WISC-IV]) alongside psychiatric examination. All of the subjects underwent otorhinolaryngological examination. Depending on examination findings, the patient's history and family history, patients at risk of hearing loss also underwent audiometric examination. Children under 5 years of age $(n=2)$ underwent play audiometry and children over 5 years $(\mathrm{n}=11)$ underwent pure tone audiometry.

The data obtained were evaluated using the SPSS 17.0 (SPSS Inc. Chicago, Illinois, USA) software application running on Windows. Descriptive data were obtained as minimum, maximum, mean, standard deviation, number and percentage. In the evaluation of normal distribution, the Kolmogorov-Smirnov test, and in the comparison of independent groups, the Student t-test, were used. A $p$ value $<0.05$ was accepted as indicating statistical significance. 


\section{Results}

Of the 64 subjects included in the study, 19 were female and 45 male. The mean age was $7.36 \pm 3.76$ years. The mean age of their mothers was $34.39 \pm 6.12$ years and the mean age of the fathers was $40.05 \pm 7.51$ years. Prenatal complications had occurred in $17.7 \%$ of cases, perinatal complications in $3.2 \%$ and postnatal complications in $25 \%$ (i.e. history of incubator use or phototherapy). The mean number of siblings was $1.98 \pm 1.49 .60 .9 \%$ of the subjects were the first child, and $23.4 \%$ the second child, of their families. The mean time interval elapsed before starting walking was $15.06 \pm 6.72$ months and the mean age of utterance of the first meaningful words was 17.48 \pm 9.26 months. The sociodemographic characteristics of the subjects are given in Table 1.

The mean age at which stuttering began was $4.85 \pm 2.34$ ( $\min 2, \max 16)$ years. The mean duration of stuttering was 2.31 years. A statistically significant difference was not found between the sexes in terms of the age at which stuttering began ( $\mathrm{p}=0.12$, independent groups t-test). In $31.3 \%$ of the subjects, a life stressor was identified which led to stuttering (Table 1).

There was a comorbid psychiatric diagnosis in $40.6 \%$ of cases. The most common comorbid psychiatric diagnoses were specific learning disorder (SLD), speech sound disorder and attention deficit hyperactivity disorder (ADHD) (Table 2). Eleven out of the 35 children who were at school age had SLD (31.4\%). 11 subjects were taking psychiatric medication. The most frequently used medications were psychostimulants and selective serotonin reuptake inhibitors. The age of onset of stuttering for subjects with a psychiatric diagnosis was $6.09 \pm 2.94$ years, whereas it was $4.13 \pm 1.55$ years for those with no psychiatric diagnosis. A statistically significant difference was determined between the two groups $(\mathrm{p}=0.001$, independent groups $t-$ test).

\section{Discussion}

The mean age when stuttering began was determined as $4.85 \pm 2.34$ years in this study. It was detected that stuttering began before 5 years of age in $68.7 \%$ of cases, at school age in 14 cases, and during adolescence in 2 cases. Developmental stuttering usually starts between the ages of 2-5, which is the period during which the most rapid development in speech occurs, and spontaneous remission occurs within 2 years in $75 \%$ of cases. ${ }^{[19]}$ Similarly to the results of our study, Morley ${ }^{[20]}$ states that the point at which stuttering begins in $85 \%$ of cases is prior to age 8 .

Stuttering is seen twice as often in males compared to females. ${ }^{[21]}$ Similarly to some other studies found in the literature, $70.3 \%$ of cases in our study were male, and the proportion of males to females was 2.3. However, it has been stated that in cases of stuttering occurring at an early age, the male-female ratio is similar, but as age increases, this ratio increases towards a male predominance. ${ }^{[4]}$ In our study, it was observed that the male/female ratio $(n=5 / 6)$ is close to unity in cases under the age of 4 . Different theories have been put forward to explain the sex ratios observed. The greater effect on males than females of life stressors, the earlier maturation of females, the possibility of hereditary transmission and the varying profiles of androgenic hormones have been cited in such theories. In a study by Selçuk et al ${ }^{[22]}$, it was determined that the testosterone level in males who stutter was higher than in a control group. The authors therefore suggest there might be a relationship between stuttering and testosterone levels.

The effect of birth complications on stuttering has also been investigated. According to the results of one review, children who suffer cerebral injury either at birth or at other times stutter more frequently than control subjects of the same age. ${ }^{[23]}$ In a large sample study, the risk factors for stuttering were ascertained as the following: perinatal complications, incubator occupancy (due to prematurity), likely attention deficit hyperactive disorder, parental alcohol misuse, a family history of obsessive compulsive disorder, maternal disability and having a foreign-born parent. ${ }^{[24]}$ In our study, only 3 cases of premature birth were found. In addition, a history of incubator occupancy and phototherapy were present in $25 \%$ of cases.

In a study comparing cases of stuttering with healthy subjects, $30-60 \%$ of the cases with stuttering had a positive family history, whereas this occurred in less than $10 \%$ of the healthy control group. ${ }^{[4]}$ In our study we found a family history of stuttering in $21.9 \%$ of cases. Avc1 et al ${ }^{[17]}$ likewise determined that $18.6 \%$ of cases had first degree relatives with a history of stuttering. The high rate of positive family history in stuttering cases has meant aetiological studies have generally focused on genetic factors, but a definite model of heredity has not yet been proposed.

Acquired stuttering almost always emerges due to neurological or psychological factors. ${ }^{[25]}$ In our study, in virtually all of the school age or adolescent cases of stuttering where there were no pre-existing problems with the fluency of speech, there was an identifiable triggering event. More specifically, starting primary school was the most frequently observed triggering factor for stuttering to occur in the school age period. Avc1 et al ${ }^{[17]}$ reported that $33.5 \%$ of the children diagnosed with stuttering had suffered a life 
Table 1. Sociodemographic characteristics of the subjects diagnosed with stuttering and variables related to stuttering

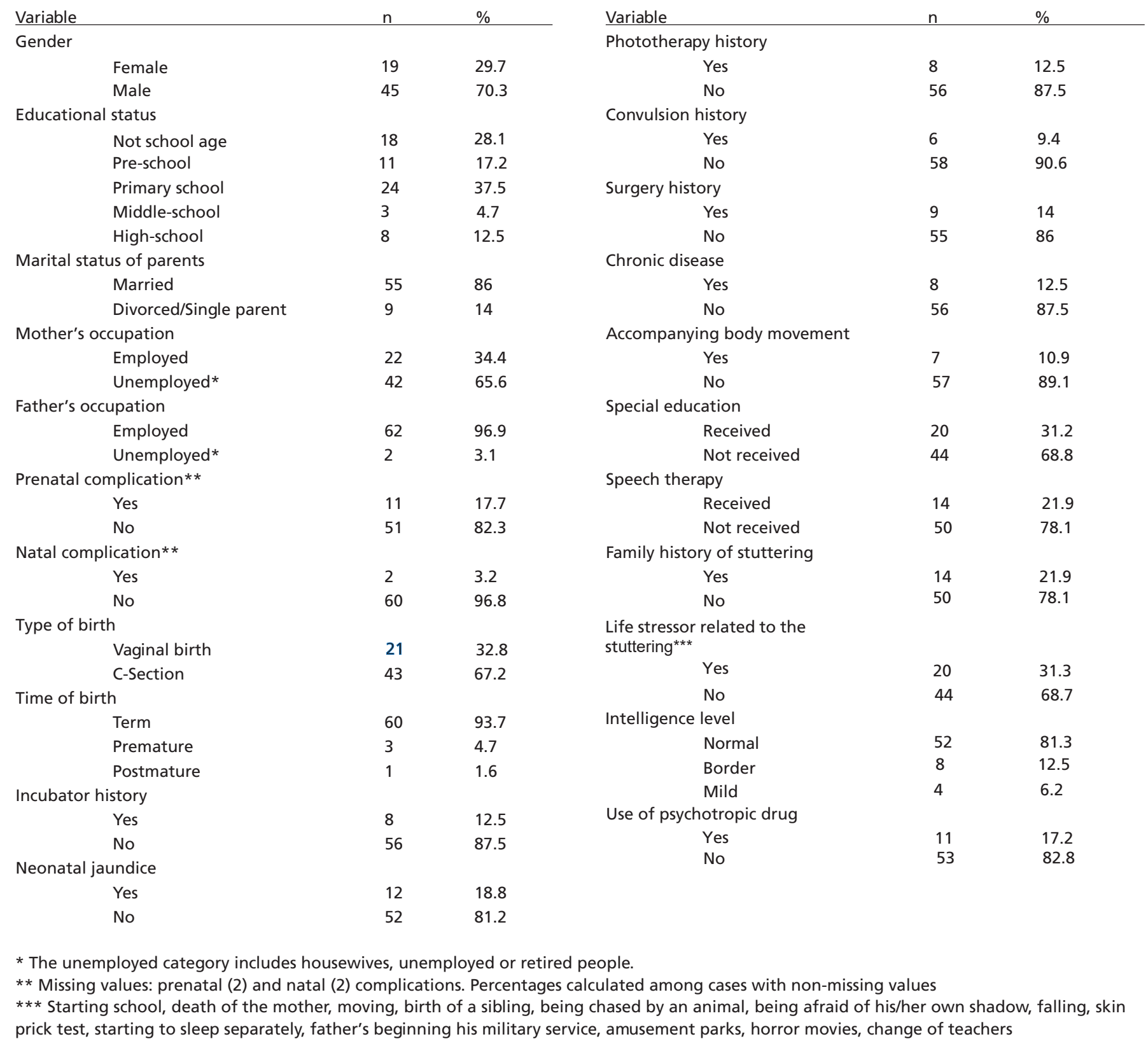

stressor at no more than one week prior to stuttering and the prognosis of stuttering which emerged after a stressor factor was positive. Similarly, we found that the rate of occurrence of a life stressor was $31.3 \%$. Life stressors can be listed as starting school, various fears, death of the mother, birth of a sibling and moving. Since our study was not a follow-up study, it was not possible to compare and evaluate the rate of spontaneous remission in these cases. It is generally accepted that stuttering cannot be explained by any single-factor theory, and the pathogenesis is thus multifactorial. ${ }^{[9]}$

In $67.1 \%$ of cases, stuttering had been present for more than 6 months. The following are risk factors for persistent stuttering: male, family history of stuttering, stuttering beginning at 3.5 years of age or older, having a comorbid language or speech disorder, stuttering severely at the age 


\begin{tabular}{lcc}
\hline Table 2. Comorbid psychiatric diagnosis. & & \\
\hline & $\mathbf{n}$ & $\%$ \\
\cline { 2 - 3 } & & \\
\hline & 12 & 18.7 \\
Attention deficit hyperactivity dis- & 6 & 9.3 \\
order & & \\
Speech sound disorder & 5 & 7.8 \\
Post-traumatic stress disorder & 2 & 3.1 \\
Generalized anxiety disorder & 2 & 3.1 \\
Social anxiety disorder & 2 & 3.1 \\
Enuresis nocturna & 1 & 1.5 \\
Autism spectrum disorder & 1 & 1.5 \\
No diagnosis & 38 & 59.4
\end{tabular}

of 4 to 5 years and early delays in the acquisition of the fundamental motor processes involved in speech. ${ }^{[1,4,26,27]}$ In our study, $74.4 \%$ of the cases whose stuttering lasted more than 6 months were male, $23.3 \%$ had a family history of stuttering, and $7 \%$ had an accompanying speech sound disorder. The age at the onset of stuttering in $76.7 \%$ of cases was 3.5 years or older. According to the normal developmental stages for speech, it is expected that a child will utter her first word at 12 months, will have 1-50 words at 18 months and be capable of 2 -word sentences at the age of $2 .{ }^{[28,29]}$ It was noted that the mean age of saying a meaningful first word for all participants diagnosed with stuttering was 17.48 months and they therefore had speech delay. Speech delay is a risk factor for the development of language-speech disorders in general. ${ }^{[30]}$

Blood et al ${ }^{[31]}$ report that articulation disorder is comorbid with stuttering in $33.5 \%$ of cases, phonological disorder in $12.7 \%$, learning difficulty in $11.4 \%$, and $\mathrm{ADHD}$ in $5.9 \%$. Arndt and Healey ${ }^{[32]}$ determined that there was a phonological and/or language disorder in $44 \%$ of children who stutter. Avc1 et al ${ }^{[17]}$ stated that in $28 \%$ of children who stuttered, a comorbid psychiatric diagnosis existed, the most frequently seen disorder being enuresis nocturna. In our study, $40.6 \%$ of cases had a comorbid psychiatric diagnosis. The most frequently encountered diagnoses were SLD, speech sound disorder and ADHD. It is noteworthy that the age at which stuttering began was higher in cases where a psychiatric diagnosis was also appropriate than in those where no such diagnosis applied. The rate of SLD was $18.7 \%$ overall, whilst in school age cases, this rate increased to $31.4 \%$. Comparing these rates with the overall prevalence of SLD in the general population, $5-15 \%$, it is evident that SLD is considerably more prevalent in individuals who stutter.[7] In one study evaluating school age subjects with stuttering, it was ascertained that the scores for attention deficit and impulsivity were raised compared to normal controls. [33] Some of the comorbidity with disorders in the neurodevelopmental group seen in stuttering subjects can be explained by shared aetiological or risk factors. Stuttering is, in any case, a neurodevelopmental disorder in its own right. In addition, it was observed that psychotropic drugs had been prescribed for comorbid psychiatric diagnoses.

This study has certain limitations, amongst which may be mentioned the fact that the intensity of stuttering was not determined using the measurement tools available. Not being able to comment on the duration of recovery and stuttering of the study participants is also a limitation, and was due to the study design not allowing for follow-up.

\section{Conclusion}

In conclusion, psychiatric diagnoses frequently accompany a diagnosis of stuttering. Children admitted to otorhinolaryngology and paediatric units with speech disorders should be evaluated by a child and adolescent psychiatrist to allow for recognition of any accompanying psychiatric disorder, and thus to allow the treatment process to be conducted more efficiently. Since SLD is frequently comorbid with childhood onset speech fluency disorder, particularly in school age children, assessment for SLD in these patients to facilitate improvement in academic performance is crucial. There is also a need to undertake preventive measures to improve the mental health of patients with stuttering.

Ethics Committee Approval: The study was received ethical approval by the Human Research Ethics Committee of Mugla Sttk1 Koçman University.

Informed Consent: Informed consent was not received due to the retrospective nature of the study

Author Contributions: Designing the study - B.G.Ö., E.Ö.; Collecting the data - B.G.Ö., E.Ö.; Analyzing the data - B.G.Ö., E.Ö.; Writing the manuscript - B.G.Ö., E.Ö.; Confirming the accuracy of the data and the analyses - B.G.Ö.

Conflict of Interest: The authors have no conflicts of interest to declare.

Financial Disclosure: The authors declared that this study has received no financial support. 


\section{References}

1. Yairi E, Ambrose NG. Early Childhood Stuttering for Clinicians by Clinicians. Austin, TX: PRO-ED; 2005. p. 521.

2. Mansson H. Childhood stuttering: Incidence and development. J Fluency Disord 2000;25:47-57.

3. McKinnon DH, McLeod S, Reilly S. The prevalence of stuttering, voice, and speech-sound disorders in primary school students in Australia. Lang Speech Hear Serv Sch 2007;38:5-15.

4. Yairi E, Ambrose NG. Epidemiology of stuttering: 21st century advances. J Fluency Disord 2013;38:66-87.

5. Boyle CA, Boulet S, Schieve LA, et al. Trends in the prevalence of developmental disabilities in US children, 1997-2008. Pediatrics 2011;127:1034-42.

6. Van Borsel J, Tetnowski JA. Fluency disorders in genetic syndromes. J Fluency Disord 2007;32:279-96.

7. American Psychiatric Association. and American Psychiatric Association. DSM-5 Task Force. Diagnostic and statistical manual of mental disorders: DSM-5. 5th ed. 2013, Washington, D.C: American Psychiatric Association; 2013. p. 947.

8. Prasse JE, Kikano GE. Stuttering: an overview. Am Fam Physician 2008;77:1271-6.

9. Smith A, Weber C. How Stuttering Develops: The Multifactorial Dynamic Pathways Theory. J Speech Lang Hear Res 2017;60:2483-505.

10. Packman A. Theory and therapy in stuttering: a complex relationship. $J$ Fluency Disord 2012;37:225-33.

11. Starkweather CW, Givens-Ackerman J. Stuttering. Austin, TX: PROED; 1997.

12. Smith A, Kelly E. Stuttering: Adynamic, multifactorial model, in Nature and treatment of stuttering: New directions. In: Curlee RF and Siegel GM, editors. Boston, MA: Allyn \& Bacon; 1997. p. 204-17.

13. Ezrati-Vinacour R, Levin I. Time estimation by adults who stutter. J Speech Lang Hear Res 2001;44:144-55.

14. Kraft SJ, Yairi E. Genetic bases of stuttering: the state of the art, 2011. Folia Phoniatr Logop 2012;64:34-47.

15. Chang SE, Erickson KI, Ambrose NG, Hasegawa-Johnson MA, Ludlow CL. Brain anatomy differences in childhood stuttering. Neuroimage 2008;39:1333-44.

16. Chang SE, Zhu DC. Neural network connectivity differences in children who stutter. Brain 2013;136:3709-26.

17. Avcı A, Uğuz Ş, Toros F. Stuttering in Children: A Comprehensive Study. [Article in Turkish] J Clin Psy 2002;5:16-21.

18. McClean MD, Runyan CM. Variations in the relative speeds of oro- facial structures with stuttering severity. J Speech Lang Hear Res 2000;43:1524-31.

19. Packman A, Code C, Onslow M. On the cause of stuttering: Integrating theory with brain and behavioral research. J Neuroling 2007;20:353-62.

20. Morley ME. The development and disorders of speech in childhood. $3 \mathrm{~d}$ ed. Baltimore: Williams and Wilkins Co; 1972. p. 542.

21. Baker BM, Blackwell PB. Identification and remediation of pediatric fluency and voice disorders. J Pediatr Health Care 2004;18:87-94.

22. Selçuk EB, Erbay LG, Özcan ÖÖ, Kartalci Ş, Batcioğlu K. Testosterone levels of children with a diagnosis of developmental stuttering. Ther Clin Risk Manag 2015;11:793-8.

23. Andrews G, Craig A, Feyer AM, Hoddinott S, Howie P, Neilson M. Stuttering: a review of research findings and theories circa 1982. J Speech Hear Disord 1983;48:226-46.

24. Ajdacic-Gross V, Vetter S, Müller M, et al. Risk factors for stuttering: a secondary analysis of a large data base. Eur Arch Psychiatry Clin Neurosci 2010;260:279-86.

25. Kaya İ. Çocuklukta Başlayan Konuşma Akıcılığı Bozukluğu (Kekemelik). In: Mukaddes NM and Ercan ES, editors. Nörogelişimsel Bozukluklar. İstanbul, Nobel Tıp Kitabevi; 2018.

26. Childhood Fluency Disorders: Overview-ASHA [cited 25.05.2019]; Available from: https://www.asha.org/practice-portal/clinical-topics/ childhood-fluency-disorders/.

27. Walsh B, Usler E, Bostian A, et al. What Are Predictors for Persistence in Childhood Stuttering? Semin Speech Lang 2018;39:299-312.

28. Çiyiltepe, M. and T. Türkbay, Speech concepts and evaluation of the child with speech delay: a review. Turk J Child Adolesc Ment Health. [Article in Turkish] 2004;11:89-97.

29. Koçyiğit M, Örtekin SG, Çakabay T, et al. Principles of approach to children with delayed speech child. [Article in Turkish] ACU Sağlık Bil Derg 2017;8:1-5

30. Mukaddes NM, Mutluer T. İletişim Bozuklukları. In: Mukaddes NM and Ercan ES, editors. Nörogelişimsel Bozukluklar. İstanbul, Nobel Tıp Kitabevi; 2018.

31. Blood GW, Ridenour VJ, Qualls CD, Hammer CS. Co-occurring disorders in children who stutter. J Commun Disord 2003;36:427-48.

32. Arndt J, Healey EC. Concomitant Disorders in School-Age Children Who Stutter. Lang Speech Hear Serv Sch 2001;32:68-78.

33. Monfrais-Pfauwadel MC, Lacombe I. Attention deficits in the school aged stuttering child: constituent trait or comorbidity. [Article in French] Rev Laryngol Otol Rhinol (Bord) 2002;123:291-5.

This is an open access article distributed under the terms of the Creative Commons Attribution-NonCommercial-NoDerivs 3.0 Unported (CC BY- NC-ND3.0) Licence (http://creativecommons.org/licenses/by-nc-nd/3.0/) which permits unrestricted noncommercial use, distribution, and reproduc- tion in any medium, provided the original work is properly cited.

Please cite this article as: Özgür BG, Özgür E. An Analysis of Sociodemographic and Clinical Characteristics in Children and Adolescents Diagnosed with Childhood Onset Speech Fluency Disorder. ENT Updates 2019;9(3): 185-190. 